\title{
Henning Prill
}

\section{Die Präsentation der Software-Sammlung Feibel}

Zusammenfassung: An der Zentral- und Landesbibliothek Berlin (ZLB) wird die Software-Sammlung des Journalisten und Autors Thomas Feibel katalogisiert und inventarisiert. Um die Computerspiele der Sammlung einer breiten Öffentlichkeit zugänglich zu machen, wurde die Gamelounge gegründet. In einer angenehmen Atmosphäre können Computerspiele der letzten 20 Jahre erlebt werden. RegelmäBige Veranstaltungen runden das Angebot der Gamelounge ab und präsentieren die Sammlung Feibel in einem öffentlichkeitswirksamen Rahmen.

Schlüsselwörter: Kindersoftware, Softwaresammlung, Computerspiel

\section{The Presentation of the Feibel Software Collection}

Abstract: The Zentral- und Landesbibliothek Berlin (ZLB) catalogs and inventories the software collection by the author and journalist Thomas Feibel. In order to make the computer games collection accessible to the public, the Gamelounge was founded in 2011. In pleasant atmosphere users have the chance to enjoy computer games of the last 20 years. Regular events complete the offer of the Gamelounge and present the Feibel collection in a good publicity environment.

Keywords: children software, software collection, computer game

Henning Prill: prill@zlb.de

Die Software-Sammlung von Thomas Feibel umfasst derzeit etwa 16000 Titel. Den Themenschwerpunkt innerhalb der Sammlung bildet die Kindersoftware. Sämtliche eingehende Medien werden zunächst von Mitarbeitern katalogisiert und inventarisiert. Dabei spielen Kriterien wie das Genre, der Medientyp oder die Altersempfehlung eine entscheidende Rolle. Mit dabei sind auch Zusatzgeräte wie Mischpulte, Lenkräder, Gesellschaftsspiele oder auch Musikinstrumente. Die Sammlung wächst stetig weiter. Über 1500 Titel kommen jedes Jahr hinzu. Bislang sind auf 27 Konsolen 3600 Spiele verfügbar.

Die Computerspiele können in der Gamelounge der „e-LernBar“ erlebt werden. Der Spezialbereich „e-LernBar“ ist ein multimediales Lernzentrum mit dem Schwerpunkt e-learning. Beide Einrichtungen sind Teil der ZLB. 


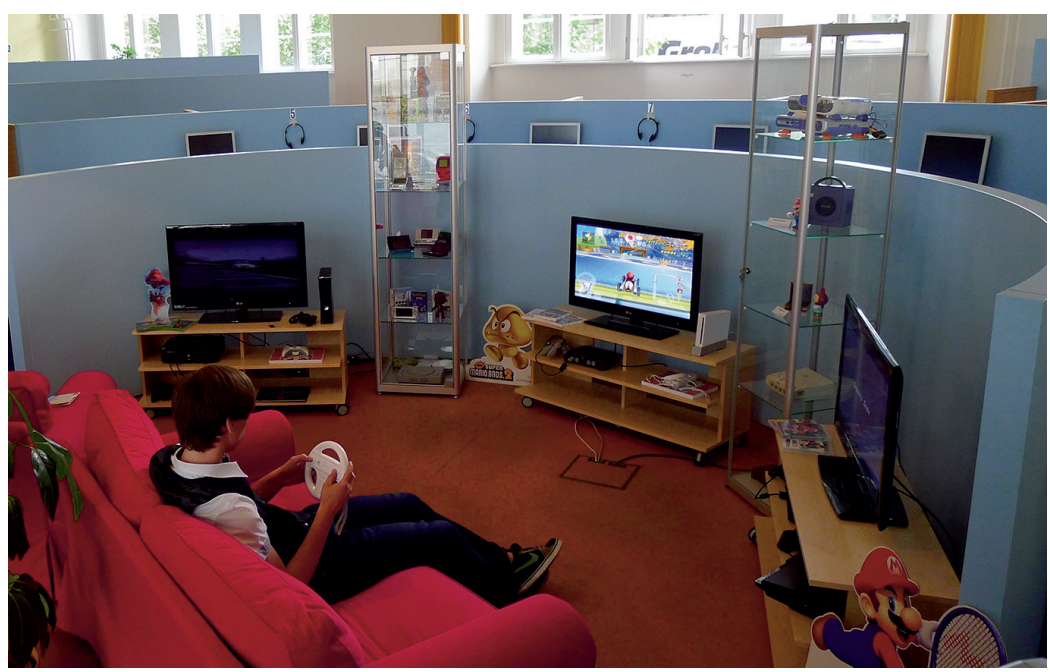

Abb. 1: Gamelounge ( Henning Prill

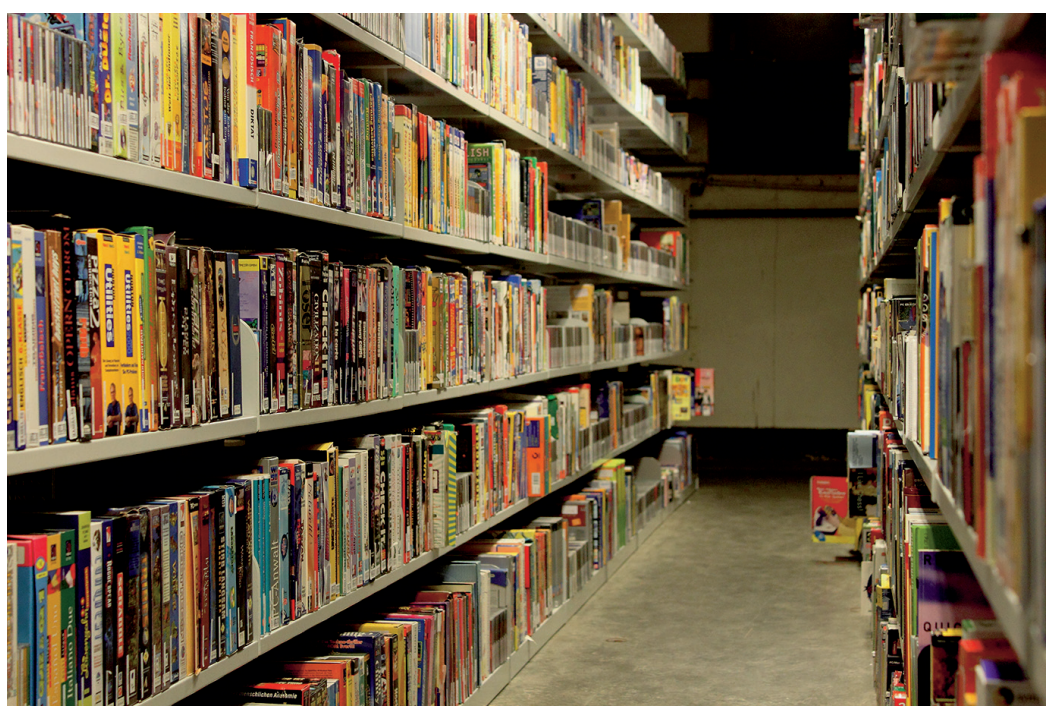

Abb. 2: Magazin @ Jana Viehweger

Untergebracht ist die Sammlung in einem separaten Magazin. Ein Katalog steht Online und vor Ort zur Verfügung. Aus Gründen des Bestandsschutzes können die Medien nicht entliehen werden. Nach der Bestellung werden die Spiele sowie die dafür benötigte Hardware in der Gamelounge bereitgestellt. Auf bequemen 
Sitzmöglichkeiten und mit Hilfe moderner technischer Ausstattung, können die Spiele in aller Ruhe getestet werden. Die Gamelounge bildet somit die Schnittstelle zwischen der Software-Sammlung und den Nutzern.

Es gab und gibt verschiedene Angebote, um die Sammlung in der Berliner Bevölkerung zu bewerben.

Dazu gehören auch Ausstellungen. 2010 thematisierte eine Ausstellung die Entwicklung von Nintendos Gameboy. Alle jemals aufgelegten Versionen des Gameboys wurden präsentiert. Auf Wunsch konnte jedes Gerät getestet werden. Das Herzstück war der Gameboy Light, der exklusiv nur in Japan vertrieben wurde.

Die Gamelounge ist auch bei unterschiedlichen Veranstaltungen innerhalb der Bibliothek präsent. Ob beim jährlichen Buchverkauf, beim Bibliothekarstag oder bei spontanen Bestandspräsentationen an den Eingangsbereichen der Bibliothek. Ab 2013 wird es in der Kinder- und Jugendbibliothek der ZLB regelmäßig Veranstaltungen zu verschiedenen Themen geben.

Passend zu sportlichen Ereignissen wie der Fußball-EM oder den Olympischen Spielen, präsentiert die Gamelounge Sportspiele aus der Software-Sammlung. Runden Geburtstagen, wie dem des Computerspiels „Zelda“ wurden ganze Ausstellungen gewidmet. Neben Texttafeln, die über die Entstehungsgeschichte dieser Adventure-Spielreihe informierten, konnten die Spiele zudem ausprobiert werden. Anhand des unterschiedlichen Veröffentlichungszeitraums der Spiele konnte die grafische Entwicklung der letzten Jahre nachvollzogen werden.

Im Herbst 2012 wurde das erste Mal die Gaming-Liga, ein deutsch-amerikanisches Computerspielturnier, in der Gamelounge ausgetragen.

Auf Nintendos Wii traten die Teilnehmer in verschiedenen Spielen gegeneinander an. Die Gewinner durften zum deutschen Endausscheid nach Wolfsburg reisen, bei dem die Sieger für das Finale in den USA bestimmt wurden.

Bislang sind ausschließlich Konsolenspiele in der „Feibel-Sammlung“ verfügbar. In Zukunft ist die Anschaffung von PCs geplant, da vermehrt Spiele und Software für die Plattform PC präsentiert werden sollen. Immerhin besteht ein Großteil der Sammlung aus PC-Medien.

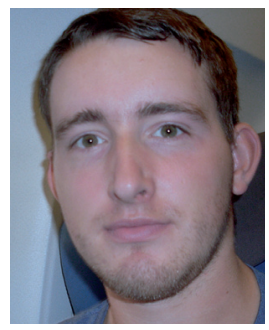

Henning Prill, Zentral- und Landesbibliothek Berlin

Breite Str. 30-36

D-10178 Berlin - Mitte

Email: prill@zlb.de 